\title{
Electrochemical gating: A method to tune and monitor the (opto)electronic properties of functional materials
}

\author{
D. Vanmaekelbergh *, A.J. Houtepen, J.J. Kelly \\ Condensed Matter and Interfaces, Debye Institute, Utrecht University, Princetonplein 1, 3508 TA Utrecht, The Netherlands \\ Received 3 November 2006; received in revised form 5 February 2007; accepted 6 February 2007 \\ Available online 20 February 2007
}

\begin{abstract}
Electrochemical polarization of a crystalline, polymeric or nanoporous system or a single molecule may change the density of charge carriers in a controlled way, and hence the optical and electrical properties. If the system has two contacts, its electronic conductivity can be measured in situ as a function of the charge carrier density that is varied by the electrochemical potential. This is called electrochemical gating. Such investigations can reveal the nature of the charge carriers (mobile or localized) and the mechanism of electronic conduction. Here, we present a brief review of a number of systems including inorganic crystals, polymers, nanoporous quantum-dot solids, and single molecules for which electrochemical gating was used successfully in the study of the electronic properties.
\end{abstract}

(C) 2007 Elsevier Ltd. All rights reserved.

Keywords: Quantum dot; Conducting polymer; Carbon nanotube; Transport properties; Change doping

\section{What is electrochemical gating?}

It is now almost 60 years since the invention of the transistor at Bell Labs. On the occasion of the 40th anniversary of this event, in 1987, John Bardeen published reminiscences of that exciting period [1]. He describes how in 1945 William Shockley had suggested making a semiconductor amplifier based on the field-effect principle. It was hoped to vary the conductance of a semiconducting thin film by a transverse electric field induced between the sample and a metal gate electrode. Initial attempts to observe the field-effect failed due to the screening of the field by charge (variations) in interfacial states. It was found that the surface-state problem could be avoided if the transverse field were applied through an electrolyte solution in contact with the semiconductor surface. Amplification of the current between source and drain electrodes was observed on varying the voltage between a metal wire in the electrolyte solution and the semiconductor electrode. Hence, the first working semiconductor transistor was based on electrochemical gating, a fact that is not generally known. Later, by growing a well-defined oxide on the germanium - from solution - the problem with the interfa-

\footnotetext{
* Corresponding author. Tel.: +31 30 2532218; fax: +31 302532403 .

E-mail address: daniel@phys.uu.nl (D. Vanmaekelbergh).
}

cial states was solved, and the all-solid-state transistor, that has revolutionized our society, was born.

The principle of electrochemical gating was, however, not abandoned. In 1984 Wrighton and co-workers reported transistors based on an active layer of polymer, that was electrochemically gated $[2,3]$. The potential between the polymer film and a reference electrode controlled the density of valence holes in the polymer, and hence the conductance of the film connecting a source and drain electrode. Later work by Hulea et al. showed that the electrochemically gated device could be used successfully as a diagnostic tool for investigating the conductance of polymers [4]. Wrighton et al. [5] also reported a gated $\mathrm{pH}$ sensor based on $\mathrm{WO}_{3}$ in which protons were intercalated in the lattice by changing the electrochemical potential of the oxide film. Electrochemical charging of a nanoporous system that consisted of $\mathrm{ZnO}$ particles was studied by Weller and coworkers [6]. In subsequent research, Meulenkamp and our group studied the electronic conductivity of nanoporous assemblies consisting of size-quantized $\mathrm{ZnO}$ nanocrystals using the electrochemical gating method [7-9]. This work has been extended to many different quantum-dot systems [10-17]. The most recent developments in the field involve electrochemically switchable single-molecule transistors based on carbon nanotubes [18-22] and organic molecules attached between a substrate and the tip of an electrochemical STM [23]. 


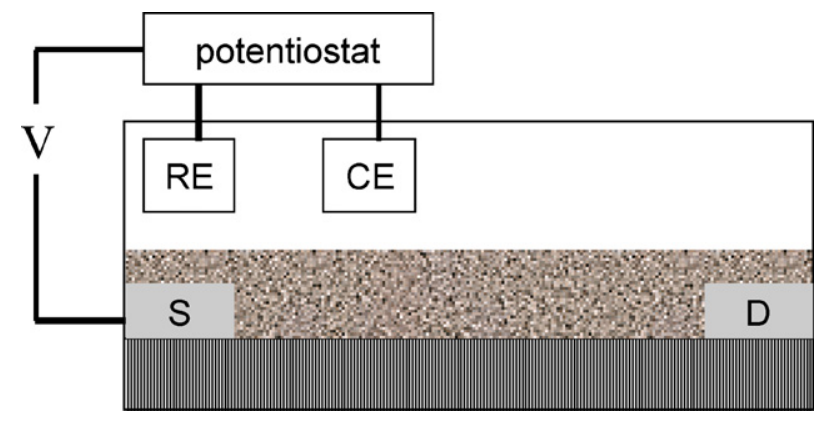

Fig. 1. Cell used for electrochemical gating of a material. A conventional threeelectrode cell is used with the material of interest as a working electrode. A potentiostat is used to apply a voltage $V$ between the reference electrode and the working electrode controlling the electrochemical potential (Fermi-level) of the material. The charge injected into the working electrode is measured in the external circuit. The electronic conductance in the linear regime of the material can be measured independently by applying a small voltage between the source (S) and drain (D) electrodes [circuit not shown]. In situ optical spectroscopy is often possible.

We first consider the general principles behind electrochemical gating. Electrochemical interfaces usually consist of a solid-phase electron conductor in contact with an ionic solution. In practice, the solid can be placed as working electrode in a conventional three-electrode electrochemical cell, its electrochemical potential is controlled by applying a voltage $V$ with respect to a reference electrode. Since the device is used to measure the electronic conductance of the material of interest (a polymer, a nanocrystal assembly, or even a single molecule) the working electrode has two electrical contacts S and D (Fig. 1). One contact is used to apply the desired voltage with respect to the reference electrode and to measure the charge injected into the system. Between the two contacts a small bias can be applied and the linear conductance can be measured independently. Measurement of the conductance of the system as a function of the charge-carrier concentration, controlled by the electrochemical potential, forms the basis of electrochemically gated devices.

The electrochemical potential of the solid phase, $\mu_{\mathrm{e}}$, can be changed by changing the voltage $V$ between (one of the contacts to) the working electrode and the reference electrode: $\mu_{\mathrm{e}}=-e V+$ constant. The interphase is located partly at the solid side and partly at the solution side, and can be described by a series connection of two capacitors with capacitances $\boldsymbol{C}_{\text {solid }}$ and $\boldsymbol{C}_{\mathrm{dl}}$, respectively. The total capacitance $C$ is given by:

$\frac{1}{C}=\frac{1}{C_{\text {solid }}}+\frac{1}{C_{\mathrm{dl}}}$

A change of the potential of the working electrode can lead to a change of the electrostatic potential drop over the electrochemical double layer-with the Fermi-level in the solid remaining unchanged with respect to the energy levels of the solid. Alternatively, a potential drop can develop across the interfacial part of the solid inducing a change of the Fermi-level in the solid with respect to the energy levels. The potential drop over the interfacial part of the solid is given by:

$$
\frac{\partial \Delta V_{\text {solid }}}{\partial V}=\frac{C_{\mathrm{dl}}}{C_{\mathrm{dl}}+C_{\text {solid }}}
$$

For the case in which $C_{\text {solid }} \gg C_{\mathrm{dl}}, \partial \Delta V_{\text {solid }} / \partial V \cong C_{\mathrm{dl}} / C_{\text {solid }} \ll 1$, a change of the electrode potential leads mainly to a change of the electrostatic potential drop across the electrochemical double layer; the charge-carrier concentration in the solid remains essentially unchanged. Consider for instance a metal electrode with an atomically flat surface exposed to the electrolyte (Fig. 2A). Increasing the electrochemical potential leads to accumulation of electrons in the very first atomic layer of the metal and ionic counter charges in the liquid part of the interphase. The study of such a double layer has been a classic subject of electrochemistry, as well as the kinetics of electron transfer between the metal phase and ions in solution. We note that if there is no suitable redox system in the electrolyte solution, electron transfer through the double layer does not occur. In electrochemistry, this is called an ideally polarizable system. We will later see that this is very similar to the solid/insulator/gate system used to change the carrier concentration in an all-solid system.

If the solid phase is a semiconductor or a molecular conductor with a low intrinsic electron concentration, we may have $C_{\text {solid }} \ll C_{\mathrm{dl}}$, so that $\partial \Delta V_{\text {solid }} / \partial V \cong 1$. This situation is depicted in Fig. 2B, in which an n-type semiconductor makes contact with an electrolyte. In the left picture, the potential of the semiconductor working electrode is chosen such that a two-dimensional interfacial layer, depleted of free electrons, is present across the solid part of the interface. Upon increasing the electrochemical potential, the Fermi-level in the interfacial layer rises with respect to the conduction band edge since $\partial \Delta V_{\text {solid }} / \partial V \cong 1$. This means that the electron concentration in the interfacial layer increases strongly (right picture). It is hence possible to change the electron concentration from almost zero (insulating state) to high values (conducting state) by electrochemical polarization, at least in the interfacial region of the semiconductor. This forms an essential element of semiconductor electrochemistry. From the point of view of the solid state, electrochemical polarization can change and control the free-carrier concentration in a two-dimensional layer of the solid [24] as in a semiconductor/insulator/gate configuration. It is obvious that by electrochemical polarization the optical properties of a semiconductor system, such as the optical absorption and reflectance, can be changed substantially. We speak of electrochemical gating when the system of interest has two contacts, such that its electronic conductance can be measured independently as a function of the electrochemical potential. The electrochemical gating principle was used rather elegantly already in 1982 by Gerischer and co-workers to study minority carrier injection from oxidised thianthrene into an n-type semiconductor singlecrystal electrode of $\mathrm{MoSe}_{2}$ [24]. In contrast to the methodology described above, the source and drain electrodes were placed in the solution close to the n-type semiconductor electrode, in a thin-layer cell. Injection of holes from the thianthrene cation gave rise to a surface inversion layer in the semiconductor, leading to a markedly enhanced conductance between the source and drain electrodes. Hence, the inversion layer acted as a shunt resistor to the solution resistance. The increase in the surface conductivity of the n-type $\mathrm{MoSe}_{2}$ electrode demonstrated that the injected holes were free and not trapped in interfacial states. 

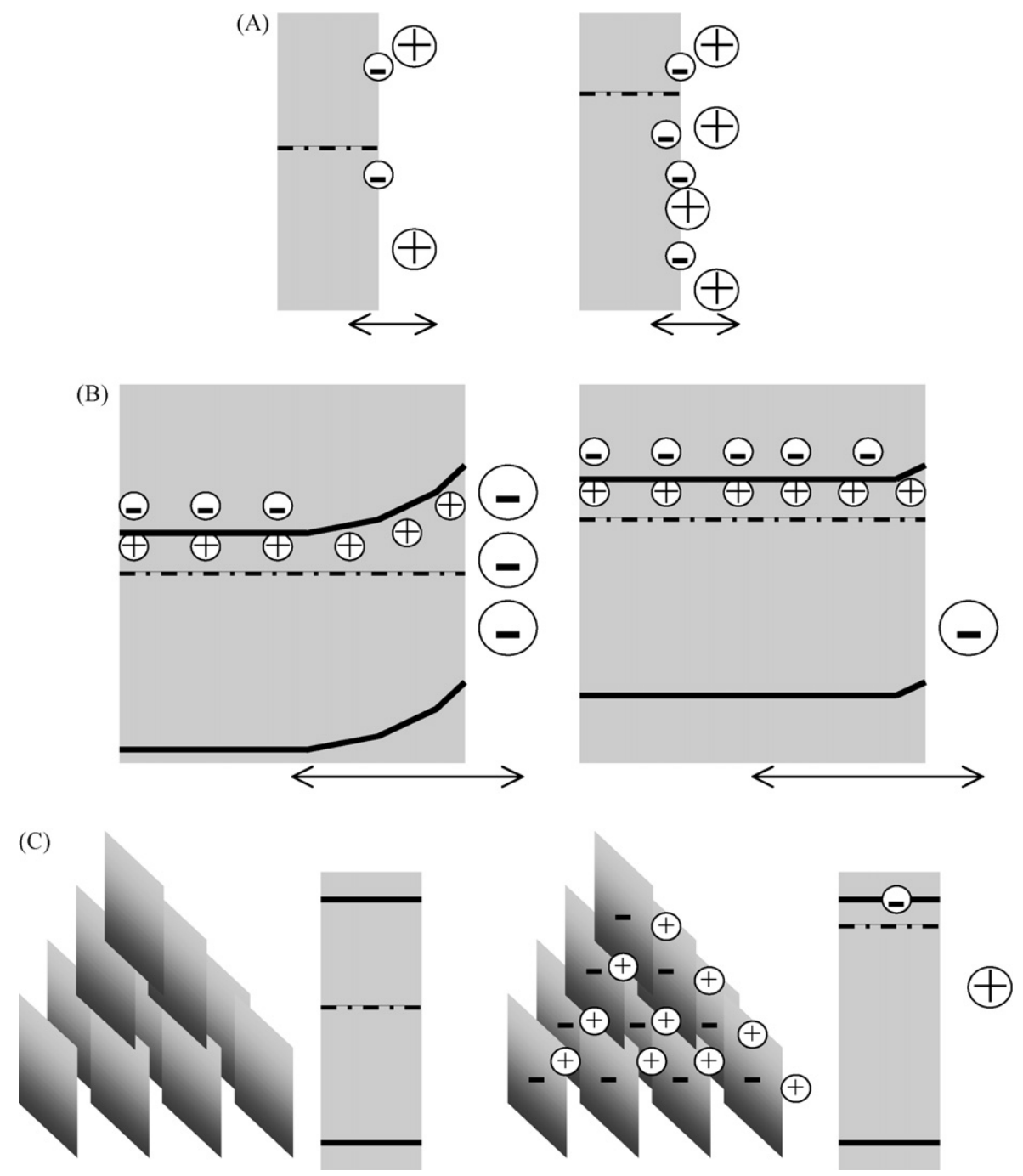

Fig. 2. Electrochemical polarization of a solid electronic conductor electrode in a concentrated electrolyte solution. (A) A metal electrode with a flat surface. Increase of the electrochemical potential of the metal working electrode leads to a change of the charge on the metal surface and the counter charge in the liquid phase, thus a variation of the electrostatic potential drop across the electrochemical double layer. The arrow indicates the width of the interphase, which is less than $1 \mathrm{~nm}$ (concentrated electrolyte solution). (B) Electrochemical polarization of a flat n-type semiconductor electrode. Left: The voltage is chosen such that there is a depletion layer for electrons at the interface. Right: Increase of the electrochemical potential leads to a change of the Fermi-level with respect to the conduction band edge and an increase of the electron concentration in the interfacial part of the solid. The total width of the interphase (arrrow) is typically $100 \mathrm{~nm}$. (C) A semiconductor system with nanometer-size voids in which an electrolyte solution can permeate. Left: System with no excess charge in the solid and liquid phase. Right: Increase of the electrochemical potential may lead to electrons occupying the LUMO of the solid phase; the charge is compensated by excess positive ions in the voids.

Another interesting example was that reported by Benisty and Chazalviel in 1993 [25] on the use of a silicon field-effect structure (p-type $\mathrm{Si}, \mathrm{n}^{+}$source and drain electrodes) with electrochemical gating to study the double-layer dynamics of a silicon/polymer electrolyte interface. They showed that the electrochemical field-effect is a superior method for investigating charge accumulation, memory effects and the influence of temperature (the glass transition) in such polymer systems.

We now turn to a nanostructured semiconductor or insulator, with pores or voids of nanometer dimensions. In Fig. 2C, left, a situation is shown in which the Fermi-level in the solid phase is midway between the HOMO and LUMO orbitals. The solid phase is hence uncharged; this also holds for the electrolyte solution in the voids of the solid that contains as many positive as negative ions. Upon increasing the electrochemical potential, the
Fermi-level in the solid phase rises with respect to the LUMO if $\partial \Delta V_{\text {solid }} / \partial V \cong 1$ (Fig. $2 \mathrm{C}$, right). Electrons accumulate in the solid phase and occupy the LUMO energy levels. Alternatively, localised states in the bandgap can become occupied. The negative charge in the solid is compensated by an excess of positive ions present in the nanovoids of the system: charge injection and compensation is truly three-dimensional. This may be compared to the two-dimensional case, described above. It is clear that in such a nanoporous system, the uptake of electrons and hence the capacitance $C \cong C_{\text {solid }}$ can be huge. When we extrapolate this to a system with "pores" of atomic dimensions we end up with an all-solid phase, where ions such as $\mathrm{H}^{+}$or $\mathrm{Li}^{+}$can intercalate in the solid lattice itself to compensate for the charge of electrons [26-28]. This type of system is used to store electrical energy in batteries. Returning to the porous system, we should realize 
that the electron concentration in the entire three-dimensional solid is controlled by the electrochemical potential. Since conditions of electrochemical (electronic) equilibrium prevail, Fermi statistics hold. If the system is electrochemically inert, electron uptake and release is completely reversible and controllable, as in a battery. It is to be expected that the electrical and optical properties of such a nanoporous system can be changed substantially by electrochemical polarization. Optical analysis can be used to determine the occupation of the conduction orbitals and sometimes localised interfacial states. On the other hand, optical effects controlled by the electrochemical potential have practical importance, e.g. in electrochromic devices [29-31].

Finally, we consider a nanoelectrode, such as a single carbon nanotube or a semiconductor nanowire. Electrochemical polarization can lead to a change of the electron concentration in the tube or wire if $\partial \Delta V_{\text {solid }} / \partial V$ is non-zero [19]. The cartoons of Fig. 2, bottom left and right can be used here too. The wire conductance can be measured between two metal contacts (screened from the electrolyte) [21,32]. This is very similar to an electrical device, in which the nanotube conductance is changed by polarizing the metal-gate/insulator/nanotube.

Here, we present a brief review of a number of systems for which electrochemical polarization has proven to be a successful method to alter the density of charge carriers in a controlled way and in which the conductivity is measured in situ. These include conducting polymers, inorganic solids with ion-intercalation, nanoporous quantum-dot solids, and single-molecule transistors.

\section{Conducting polymers}

The era of conducting polymers started in 1977 when Heeger, Mac Diarmid and co-workers discovered that the electronic conductivity of polyacetylene could be increased markedly by chemical oxidation $[33,34]$. Since then, many polymer systems have been investigated, like polyaniline, polypyrrole, and polythiophene. This work has led to numerous applications in the fields of rechargeable batteries, electronic and electrochromic devices ("plastic electronics"), LED's and solar cells and biological and chemical sensors [35-37]. In such systems the electrical properties of the conducting polymer are important. Conductivity is generally measured "ex situ", after chemical or electrochemical charging. Such an approach may be awkward for air-sensitive systems. A gated transistor geometry allows one to electrodeposit the polymer, to alter its charged state and, at the same time, to measure "in situ" the conductivity as a function of potential $[2-4,38]$. In addition, since the density of free carriers (generally valence holes) in the polymer can be deduced from the charge injected, the carrier mobility can be obtained.

In 1984, Wrighton and co-workers proposed the use of a micro-electrode array, derivatised with a conducting polymer, to mimic the characteristics of the solid-state transistor with a view to micro-sensor applications $[2,3]$. Their first publications describe what they call a "chemical transistor", a set of three gold microelectrodes (drain, gate and source) covered with polypyrrole. The electrodes were provided with controlled amounts of polymer by electrochemical deposition from a monomer solu- tion in acetonitrile. Since polypyrrole exhibits a sharp change in conductivity upon oxidation, the current passing between source and drain becomes measurable when the gate voltage is above a threshold value corresponding to the onset of oxidation. At negative potential, with the polymer in its insulating state, the device is switched off. In contrast to polypyrrole, polyaniline exhibited a maximum in the film conductance at a potential of
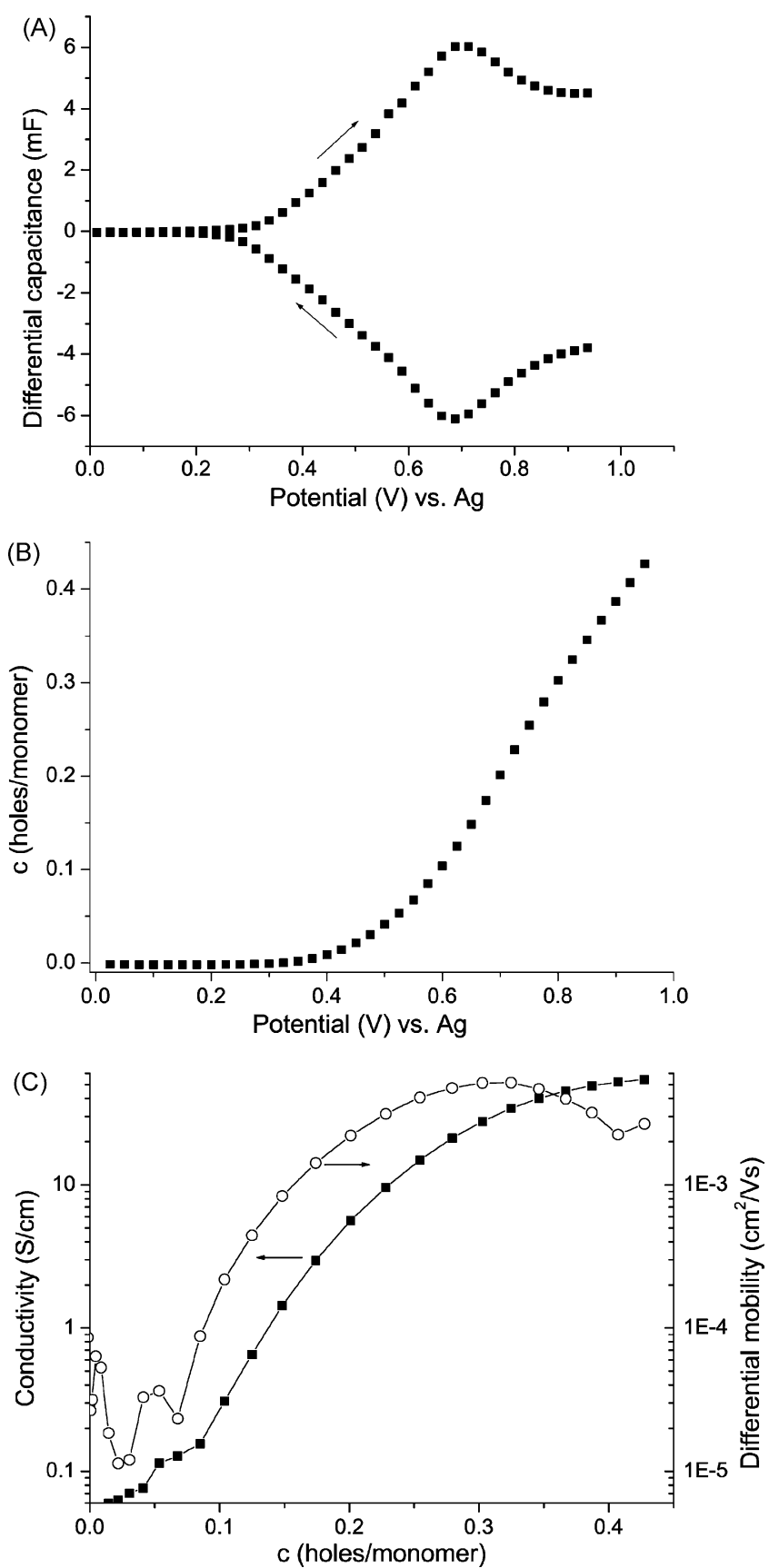

Fig. 3. Electrochemical injection of holes into a PPV film in an acetonitrile electrolyte solution. (A) The differential capacitance measured by applying successive $25 \mathrm{mV}$ steps to the film. The potential is defined with respect to a $\mathrm{Ag}$ pseudoreference electrode. The arrows show the two scanning directions. The symmetry in the charging and discharging curves shows the reversibility of charging in this system. (B) Doping (number of holes) per monomer $c\left(\mu_{\mathrm{e}}\right)$ calculated from the data in (A). (C) The dependence of the film conductivity on the doping density. The carrier mobility was obtained from the conductivity. 
of $+0.4 \mathrm{~V}$ versus SCE. Changes in resistance of polymer films on electrochemical switching can be very large, up to $10^{6}$ in the case of polyaniline [38]. In the absence of oxygen, such devices were stable for several days. As one would expect for an electrochemically based device involving significant changes in stored charge, switching times are relatively long, in the milliseconds to seconds range. However, this is not a real problem for applications involving sensing, e.g. of $\mathrm{pH}$ and redox systems.

PPV, poly(p-phenylene vinylene), is perhaps the most important material for current state-of-the-art polymer-based (opto)electronic systems. Charge transport in such a disordered conjugated system usually proceeds via thermally activated hopping. In this process, the energy-dependent density-of-states (DOS) and the charge mobility are two key parameters. Hulea et al. [4] showed that the electrochemically gated transistor can be used to achieve controlled and reversible doping of the polymer (Fig. 3A) and thus to measure the DOS function in a broad energy range (Fig. 3B) and, at the same time via the source-drain conductance, the hole mobility in a wide concentration range (Fig. 3C). This work helped to resolve a controversy regarding the DOS: it was shown that the core of the valence band DOS function is a Gaussian, while the low-energy tail has a more complex structure. The conductivity was found to increase by five orders of magnitude as the doping was increased from about 0.02 to 0.3 holes/monomer. The results confirmed that the hole mobility is concentration, or rather energy dependent. The mobilities observed in an electrochemically gated device are smaller than those observed in a classic all-solid-state field-effect transistor at similar doping levels; this is, perhaps, due to the presence of anions in the electrochemically gated polymer film that tend to localize the holes. We found recently that electrochemical gating of polymer films works down to very low temperatures, allowing us to determine the valence DOS with a better energy resolution and the temperature-dependence of the hole mobility.

\section{Intercalation in inorganic solids}

Again it was Wrighton and his team who first used the transistor geometry for an electrochemical gating reaction involving ion intercalation in an inorganic solid [5]. An array of closely spaced $\mathrm{Au}$ or Pt microelectrodes were coated with a $150 \mathrm{~nm}$ thick $\mathrm{WO}_{3}$ layer by sputtering. $\mathrm{WO}_{3}$ becomes a good conductor on electrochemical reduction in aqueous solution as a result of the reversible proton-dependent redox reaction:

$\mathrm{WO}_{3}+n \mathrm{H}^{+}+n \mathrm{e}^{-} \Leftrightarrow \mathrm{H}_{n} \mathrm{WO}_{3}$

Protons are inserted into the lattice of $\mathrm{WO}_{3}$ to compensate for the electrons, which occupy the conduction band at room temperature. The variation in resistance of the $\mathrm{WO}_{3}$ layer between source and drain electrodes spanned four orders of magnitude upon raising the electrochemical potential of the $\mathrm{WO}_{3}$ working electrode. Because of the $\mathrm{pH}$ dependence of the reaction, the electrochemical transistor could be used as a real-time $\mathrm{pH}$ microsensor: a fast and reproducible change in source-drain current was observed at a fixed electrochemical (gate) potential when solutions of different $\mathrm{pH}$ were flowed past the electrode.
In the field of rechargeable batteries $\mathrm{Li}^{+}$ion intercalation in solids (a reaction similar to the proton reaction above) is important $[27,28]$. The transistor geometry has been used by various groups to investigate lithium intercalation. In the study of $\mathrm{TiO}_{2}$ by van der Krol et al. the combination of in situ electrical and optical measurements (the system is electrochromic) was particularly effective in elucidating the electronic properties $[26,28,39]$. Three different regimes could be distinguished. The double-layer regime is characterized by injection of electrons into $\mathrm{TiO}_{2}$ leading to the formation of an accumulation layer. That the injected electrons are free, or nearly free, is clear from the large increase in conductivity and the steady increase of light absorption with increasing wavelength. In the second regime, a phase transformation to $\mathrm{Li}_{0.5} \mathrm{TiO}_{2}$ takes place and the conductance of the film decreases. In a companion study, phase changes in the system were followed with in situ X-ray diffraction [40]. The authors attribute the low conductivity in $\mathrm{Li}_{0.5} \mathrm{TiO}_{2}$ to electron repulsion effects. These changes in conductivity are accompanied by marked changes in the optical properties: visual coloration of the film is observed. In the third regime, $\mathrm{Li}$ is inserted into $\mathrm{Li}_{0.5} \mathrm{TiO}_{2}$. An increase in the conductivity indicates filling of a higher band with good conduction properties. In the same study, results are also given for intercalation in mesoporous $\mathrm{TiO}_{2}$ and the same general trends were found. However, the absolute conductivity values for the mesoporous films were much smaller than those of the dense films. Possible reasons for the reduced conductivity suggested by the authors are: percolation effects in the mesoporous structure, the presence of energy barriers for inter-particle electron transport and surface defects.

An interesting application of the "gating" principle was reported by Kooij et al. for the study of metal hydrides [41,42]. Various trivalent rare-earth metals (including yttrium and lanthanum) on reacting with hydrogen show a metal-to-insulator transition [43,44]. This transition, also observed in certain alloys [42], is accompanied by spectacular changes in the optical and electrical properties of the system. For example, formation of the $\gamma-\mathrm{YH}_{3}$ phase following exposure of an $\mathrm{Y}$ thin film to $\mathrm{H}_{2}$ in the gas phase, gives rise to optical transparency and a marked increase in the electrical resistivity of the thin film. Such changes are reversible. An interesting feature of these systems is that the transition between the reflecting metallic and transparent insulating states can be driven electrochemically, opening the way to important applications $[43,44]$. The electrolytic reduction of water can result in the hydrogenation of the metal film:

$\mathrm{H}_{2} \mathrm{O}(1)+\mathrm{e}^{-} \Leftrightarrow \mathrm{H}_{\mathrm{ads}}+\mathrm{OH}^{-}, \quad \mathrm{M}(\mathrm{s})+n \mathrm{H}_{\mathrm{ads}} \Leftrightarrow \mathrm{MH}_{n}(\mathrm{~s})$

Since the evolution of electrical and optical properties with increasing hydrogen content may be quite complex (these are multi-phase systems) Kooij et al. developed a method for the in situ determination of the film resistivity [41]. The electrochemical measurements were used to establish the hydrogen content of the film (and pressure composition isotherms). In this way the dependence of the film resistivity on hydrogen content (controlled by the electrochemical potential) could be 
measured readily in a wide range. Simultaneously, the optical properties were investigated with transmission and reflection spectroscopy. This combination of in situ electrochemical, electrical and optical methods proved very effective in monitoring and interpreting the optoelectronic properties of the metal/metal hydride "switchable mirror".

\section{Quantum-dot solids with charge-tunable optical and electrical properties}

Colloidal nanocrystals made from semiconducting III-V, II-VI, and IV-VI compounds such as InP, InAs, $\mathrm{ZnO}, \mathrm{CdS}$, $\mathrm{CdSe}, \mathrm{CdTe}$, and $\mathrm{PbSe}, \mathrm{PbTe}$ and $\mathrm{PbS}$ have attracted enormous interest in the last two decades [45-47]. Due to confinement of the electron and hole particle waves in the nanocrystal host, the electron and hole energy levels are discrete [48-50]. For instance, for the electron levels in wurtzite and zincblende nanocrystals, the LUMO has S-type symmetry and can accommodate two electrons. There are three P-type energy levels that can accommodate six electrons in total. For nanocrystal hosts below $10 \mathrm{~nm}$, the regime of strong confinement holds: the separation between the $\mathrm{S}$ and $\mathrm{P}$ energy levels increases with decreasing size of the crystal and can be between 50 and $500 \mathrm{meV}$. Similar values hold for the repulsion energy between two electrons in a nanocrystal. The optical gap between HOMO and LUMO is also strongly size-dependent due to quantum confinement. This field of nanoscience has attracted much interest since colloidal quantum-dot systems can be prepared with a bright and size-tunable exciton luminescence. In addition, colloidal quantum dots are considered as building blocks for novel types of solids.

Fig. 4A shows an array of $6 \mathrm{~nm}$ PbSe nanocrystals obtained by simple drop casting of a colloidal solution and drying. Due to the fact that the nanocrystals have the same size and shape, ordered superlattices are formed [51]. Such a superlattice can be considered as an fcc arrangement of (nearly) spherical nanocrystal building blocks, with octahedral and tetrahedral holes. In a close-packed array, the octahedral and tetrahedral holes have a size of 0.414 and 0.225 times the nanocrystal radius, while the connections between these voids are even smaller.
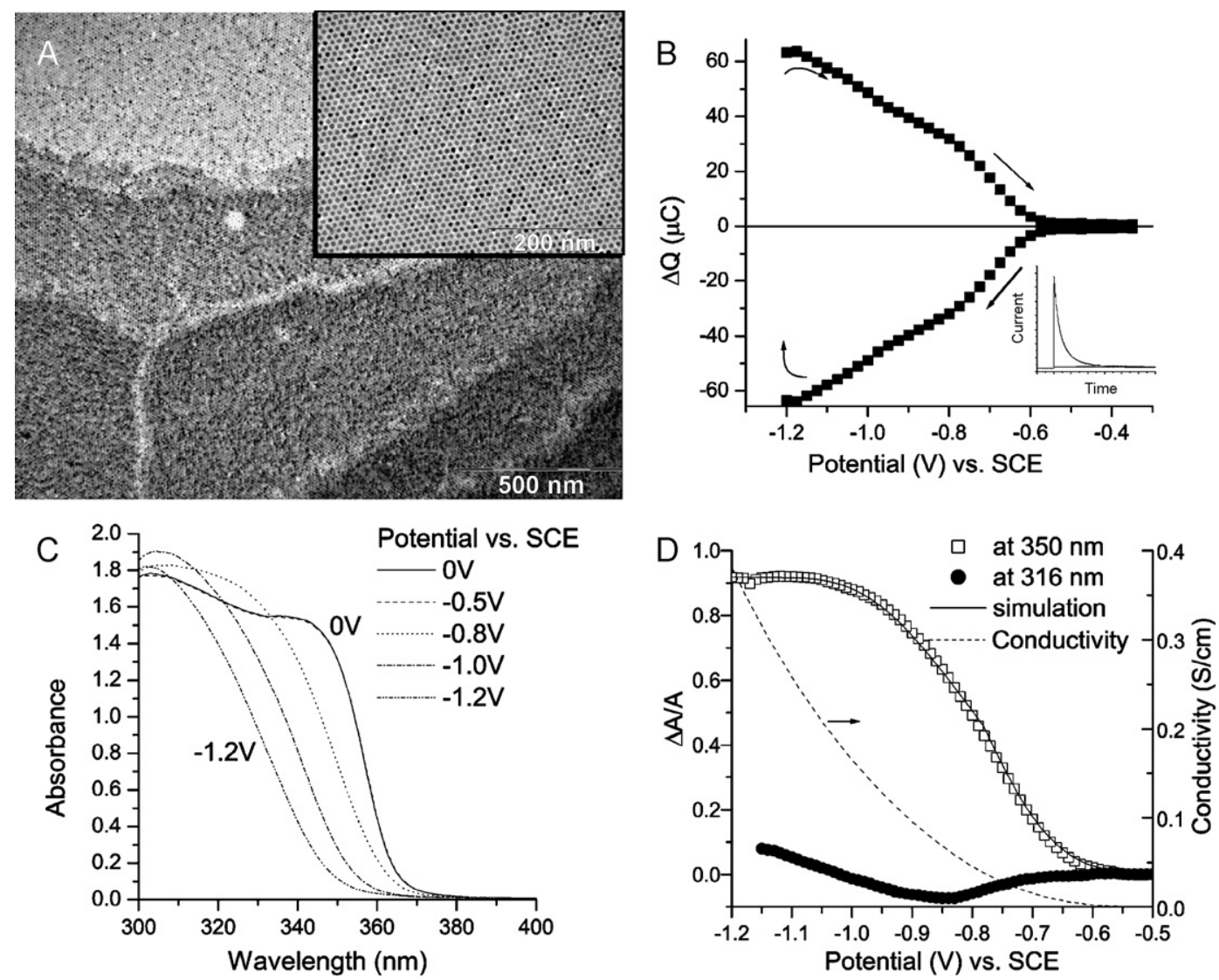

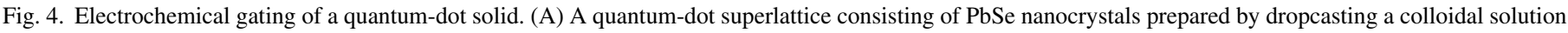

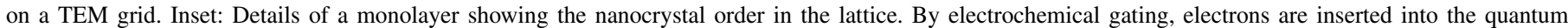

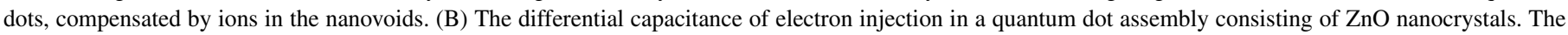

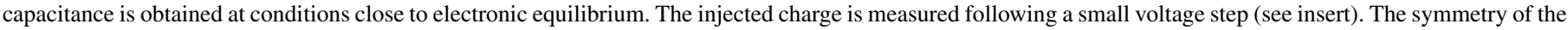

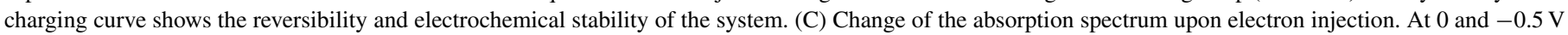

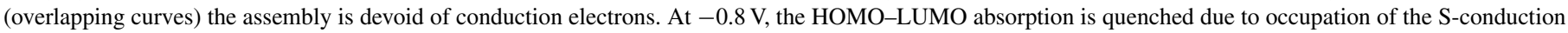

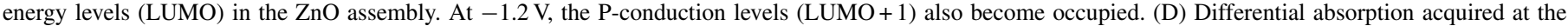

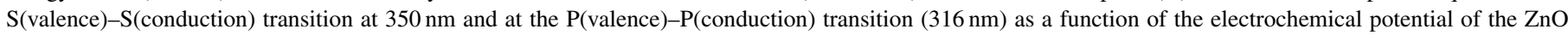

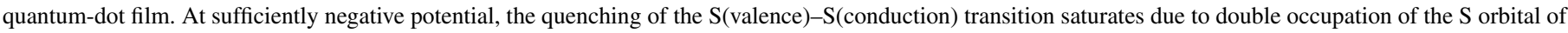
the quantum-dot film. The conductance of the film rises as soon as electrons are injected into the S orbitals of the $\mathrm{ZnO}$ quantum-dot solid. 
It is of great interest to study quantum-dot superlattices, in which the orbitals are occupied by holes and electrons. Simple optical excitation leads to electrons and holes that recombine. Alternatively, electrons can be inserted into the quantum-dot solid by strongly reducing agents, such as Na-derivates [10]. $\mathrm{Na}$ injects an electron into the quantum-dot array, and is itself inserted in the pores of the array, this corresponds to chemical gating. This leads to a certain electron occupation, but this process of chemical doping is very difficult to control. The most convenient way to insert charge carriers in a quantum-dot solid is by electrochemical gating (see Fig. 1). The charge of the electrons injected into the quantum dots is compensated by (an excess of) positive ions in the octahedral and tetrahedral holes. It is obvious that these holes must be interconnected to allow for three-dimensional charge insertion. Consider for example a model case of a perfect fcc quantum-dot lattice with one electron per quantum dot, each electron compensated by an ion in an octahedral hole. This charge-ordered system mimics a rocksalt ionic lattice; the electrostatic stabilization energy of each electron would then be described by an equation similar to that of the lattice energy, i.e. $E_{ \pm}=-e^{2} / 4 \pi \varepsilon \varepsilon_{\mathrm{o}} r_{\mathrm{QD}}\left(r_{\mathrm{QD}}\right.$ is the radius of the quantum dot, $M$ the Madelung constant typical for the lattice). This stabilization energy makes it possible to incorporate up to eight electrons per nanocrystal quantum dot in the potential window in which a system is chemically stable [9].

Fig. 4B shows the differential capacitance as a function of the electrochemical potential for electron injection into an assembly consisting of $\mathrm{ZnO}$ quantum dots. Negative of the onset, charge carriers are injected. It can be seen that the $C$ versus $V$ diagram is almost symmetrical: the charges inserted can also be extracted. This shows that the system is chemically stable. An asymmetry in the $C$ versus $V$ curve indicates the loss of charge carriers by irreversible electrochemical processes, i.e. reduction of impurities in the electrolyte solution or the semiconductor nanocrystal itself. It is possible to measure the number of quantum dots in the assembly from the number of $\mathrm{ZnO}$ molecules present (determined by chemical analysis) and the nanocrystal size. This allowed us to calculate the average number of charge carriers per quantum dot as a function of the electrochemical potential. Optical absorption spectroscopy is, however, a more direct way to probe the occupation of the quantum-dot orbitals by electrons (holes). In fact, intra-band S-P, P-D, ... transitions, lying in the near IR, can be probed [14,52]. Alternatively, the quenching of the inter-band absorption upon occupation of the electron orbitals can be measured as a direct and quantitative way to monitor the orbital occupation $[12,17,53]$. The changes in the optical absorption spectrum of a $\mathrm{ZnO}$ quantum-dot solid upon electron insertion are shown in Fig. 4C. It is clear that as soon as electrons are inserted into the system, the $\mathrm{S}(\mathrm{VB})-\mathrm{S}(\mathrm{CB})$ excitonic transition starts to become quenched, showing that the inserted electrons occupy the delocalized S orbital. The S-S quenching saturates at more negative potentials indicating double occupation of the orbital. Fig. 4D shows the fractional occupation of the $\mathrm{S}$ orbitals as a function of the electrochemical potential. This result can be understood by a model that accounts for the confinement energy, and the electron-electron repulsion within a quantum dot and between adjacent quantum dots (full line).
In this model, the positive ions inserted in the lattice and the permeating electrolyte are accounted for by an effective dielectric constant screening the repulsion of the electrons. There is a good agreement between the experimental charging curves and the results obtained by this model. The effective dielectric constant varies strongly with the nature of the permeated electrolyte: about 300 for an aqueous electrolyte and about 20 for an electrolyte in tetrahydrofuran.

Monitoring the absorption quenching, together with the differential capacitance also allows one to distinguish occupation of localized states in the bandgap from occupation of the S, P quantum-dot orbitals [17]. For instance, it became clear that solids based on CdSe quantum dots have a small, but measurable number of impurity energy levels that can capture an electron. These levels are perhaps related to the presence of residual oxygen molecules in the quantum-dot sample.

By electrochemical polarization the charge carrier concentration in a quantum-dot solid can be controlled in a three-dimensional system. It is clear that this is of key importance in the understanding of the electrical properties of such a system. The electronic conductivity and electron mobility of quantum-dot solids has been studied in detail as a function of the electronic occupation using the source-drain geometry (see Fig. 1). The systems that were investigated consisted of $\mathrm{ZnO}$ [9], $\mathrm{CdSe}$ [12] and $\mathrm{PbSe}$ [13] quantum dots. The linear conductance was measured as a function of the orbital occupation controlled by the electrochemical potential. This allows different conduction regimes to be distinguished, e.g. conduction via $\mathrm{S}$ orbitals or via $\mathrm{P}$ orbitals. A recent trend is to charge the quantum-dot solid to a certain degree, and then freeze it [54]. In this way, the temperature dependence of the conductance can be studied in the entire range between $0 \mathrm{~K}$ and the freezing point of the liquid permeating the pores.

It is also interesting to note that the electrical properties of such quantum-dot solids can be changed substantially by chemical treatment. Talapin et al. [55] reported that initially poorly conducting $\mathrm{PbSe}$ superlattices could be chemically activated by a hydrazine treatment to form $\mathrm{n}$ - or p-channel field-effect transistors with striking properties: electron and hole mobilities of 0.9 and $0.2 \mathrm{~cm}^{2} / \mathrm{V} \mathrm{s}$, respectively; current modulation with a factor $10^{3}$ to $10^{4}$ and operation at a high current density $\left(3 \times 10^{4} \mathrm{~A} / \mathrm{cm}^{2}\right)$. The chemical treatment influences the inter-particle spacing and the electronic coupling between the quantum dots, while providing doping and passivation of electronic traps.

\section{Single-molecule electrochemical devices}

The development and use of micrometer-sized electrodes has long been a subject of research in electrochemistry. For instance, the transport of a redox species to a micro-electrode becomes three-dimensional if the dimensions of the electrode are smaller than the width of the diffusion layer. The much faster diffusion enables one to study fast electrochemical kinetics. One of the aims of nanoscience is to miniaturize optical and electrical devices; this trend is having an impact on current electrochemical research $[18,21]$. The question is: can we make an 
(A)

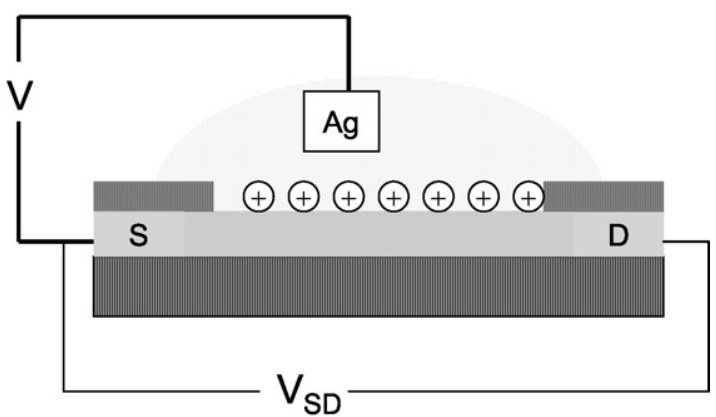

(B)

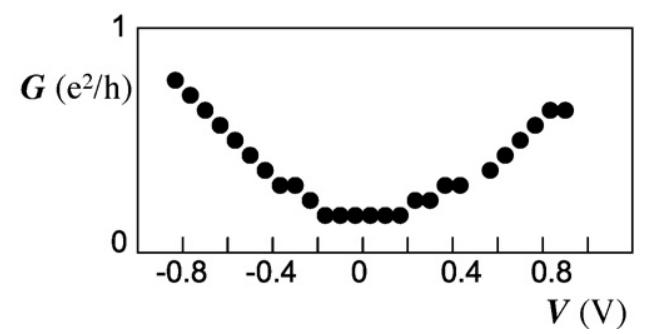

Fig. 5. Electrochemical gating of a single-walled carbon nanotube. (A) A voltage $V$ is applied between a pseudo-reference electrode consisting of a $\mathrm{Ag}$ wire and the source contact to the nanotube. Either conduction electrons or valence holes can be injected into the tube depending on the electrochemical potential. (B) The electronic conductance measured between the source (S) and drain (D) electrodes of a nanotube of length of $2 \mu \mathrm{m}$ and diameter of $4.3 \mathrm{~nm}$ is presented in the lower figure. Electrons (at negative $V$ ) and holes (at more positive $V$ ) lead to a wire conductance close to $e^{2} / h$ ( $e$ is the elementary charge, $h$ is the Planck's constant) [after Rosenblatt et al. [21]].

electrochemical electrode based on one single molecule? This challenging question has sparked the interest of leading groups in nanoscience. Nanoelectrochemistry is becoming a promising discipline which will deal with fundamental issues. For instance, it will become possible to study the electrochemical statistics of a few or even a single redox-molecule exchanging electrons with a nanometer-sized electrode. Links to the field of bioelectrochemistry and electrochemical sensing are obvious.

At present, single metal or semiconductor wires can be used as electrochemical nanoelectrodes and sensors. An electrode based on an individual, single-walled carbon nanotube (CNT) can be considered as the most prominent example of a truly single-molecule electrode. Due to their remarkable aspect ratio, carbon nanotubes are easy to contact, allowing for two separate contacts acting as a source and drain (Fig. 5). In addition, a chemically well-defined and clean surface can be exposed to the electrolyte solution. Carbon nanotubes are mechanically strong and chemically almost inert. They may be metallic or semiconducting; the density of electronic states is that of a onedimensional system, with sharp peaks on both sides of the gap known as the "van Hove singularities" [19]. The total capacitance $C$ of a CNT electrode is given by an equation similar to (1):

$C=\frac{C_{\mathrm{dl}} C_{\mathrm{q}}}{C_{\mathrm{dl}}+C_{\mathrm{q}}}$

with $C_{\mathrm{dl}}$ being the capacitance of the electrochemical double layer, and $C_{\mathrm{q}}$ the capacitance related to the occupancy of the electronic states of the carbon nanotube. The latter is often called the quantum capacitance. Since the electronic density of states of a carbon nanotube is not very large, the value of $C_{\mathrm{q}}$ can be smaller than that of $C_{\mathrm{dl}}$. When $C_{\mathrm{q}}<C_{\mathrm{dl}}$, the Fermi-level moves across the energy levels of the nanotube upon electrochemical polarization (see above and Fig. 2C). The measured total capacitance $C(V)$ then reflects the density of electronic states. $C_{\mathrm{q}}<C_{\mathrm{dl}}$ has another consequence: in electron transfer kinetics, both the density of states of the redox system (as expressed by the Marcus-Gerischer model) and those of the carbon nanotube are important for understanding the rate of resonant electron tunneling [19]. In contrast, for a typical metal electrode, only the density of states of the redox system located at the metal Fermi-level varies upon changing the voltage.

Due to their shape, carbon nanotubes are ideal molecules for field-effect transistors. For a conventional field-effect transistor with a back gate, the capacitance of the gate/oxide/CNT junction is given by an equation similar to (5) with $\boldsymbol{C}_{\mathrm{dl}}$ replaced by $\boldsymbol{C}_{\text {backgate }}$. Since $C_{\text {backgate }}$ (being about $3 \times 10^{-11} \mathrm{~F} / \mathrm{m}$ ) is much smaller than $C_{\mathrm{q}}$, the gate voltage drops mostly over the oxide layer. As a consequence large gate voltages ( $10 \mathrm{~V}$ or more) have to be used to change the electron (hole) concentration in the carbon nanotube. However, in the case of an electrochemical gate (Fig. 5), Eq. (5) should be considered. The double layer capacitance (about $10^{-8} \mathrm{~F} / \mathrm{m}$ ) is larger than the capacitance of the tube and, hence, changing the potential of the carbon nanotube leads to a change of the occupancy of the conduction electron or valence hole states of the tube. In other words, electrochemical gating is extremely efficient [21]. In Fig. 5B, the linear conductance of the carbon nanotube is presented as a function of the electrochemical potential of the tube. It is clear that, at negative potentials, electrons occupy conduction levels and lead to a large conductance, close to the quantum conductance $e^{2} / h$ ( $e$ is the electronic charge, and $h$ is the Planck's constant). At positive potentials, holes occupy the valence levels, and a similar conductance is measured. Since the density of electrons (holes) is known from the injected charge and the nanotube dimensions, the mobility of electrons or holes in the nanotube can be inferred. Values in the range of $1000-4000 \mathrm{~cm}^{2} / \mathrm{V} \mathrm{s}$, close to the ballistic limit, have been reported [21]. The large change of the conductance with potential suggests that electrochemically gated nanotubes can form the basis for sensing molecules with a charged moiety or a dipole moment. Upon attachment of such a molecule to the (modified) carbon nanotube surface, the charge configuration changes and the field affects the charge carrier density in the tube. This sensor device can be considered as a "nano-version" of the traditional ChemFET. We remark here that, regarding the motion of charge carriers so close to an interface, electrostatic interaction with molecules, i.e. surfacepolaron formation, cannot be excluded. A recent study with a molecular crystal has shown that the mobility of the carriers strongly depends on the dielectric constant of the medium [56].

Gittins et al. [23] reported another example of a singlemolecule device that can be switched on and off by electrochemical gating. Organic molecules that contain a bipyridinium group in the carbon chain are chemically attached to a gold surface on one side, and to a gold nanoparticle on the 
other side. This system is used as a working electrode in an electrochemical cell. The conductance through a single organic molecule is measured by scanning tunneling spectroscopy under electrochemical conditions, placing the STM tip above a gold particle. It is found that the conductance through the molecule depends strongly on the electrochemical potential of the gold electrode that controls the redox state of the bipyridinium group. Only when the monovalent radical ion bipy ${ }^{\bullet+}$ is present the conductance is high, it is markedly lower if the bipy ${ }^{\bullet+}$ is oxidized to bipy ${ }^{2+}$ or reduced to bipy ${ }^{0}$.

\section{Conclusions}

The examples described in this review demonstrate the power of electrochemical gating. By varying the electrochemical potential one can change the electronic properties (carrier density, mobility, etc.) of a variety of systems in a wide range and use a source-drain conductance measurement to study such changes. The method is particularly effective when combined with optical measurements: gating obviously also changes the optical properties. For example, in the case of quantum-dot solids the electrochemical potential regulates the occupation of the discrete energy levels, thus turning the optical transitions "on" or "off".

The study of the optical properties of quantum-dot solids is still at an early stage. It is obvious that fundamental aspect such as charge-exciton interaction [17], exciton Auger recombination and low-threshold lasing [56] can be studied in a controlled way. Since the charged state can be "frozen-in" the electronic and optical properties of gated solids can be investigated in a broad temperature range. This possibility will very likely find wider application in the future.

An interesting variation on the approach described in this paper for certain macroscopic systems might be an in situ Hall effect measurement. In combination with the electrochemical result (injected charge) and conductance (product of carrier concentration and mobility) the Hall effect could give extra important information.

The most interesting electrochemical challenge is, perhaps, the study of single-molecule systems. The carbon nanotube is an exciting topic for electrochemical research. Electrochemical gating of semiconductor nanowires showing size quantization should be investigated. This field will become even more relevant as nanolithography, needed to prepare well-defined systems, becomes available.

\section{References}

[1] J. Bardeen, Solid State Technol. 69 (1987).

[2] H.S. White, G.P. Kittlesen, M.S. Wrighton, J. Am. Chem. Soc. 106 (1984) 5375.

[3] G.P. Kittlesen, H.S. White, M.S. Wrighton, J. Am. Chem. Soc. 106 (1984) 7389.

[4] I.N. Hulea, H.B. Brom, A.J. Houtepen, et al., Phys. Rev. Lett. 93 (2004) $166601 / 1$.

[5] M.J. Natan, T.E. Mallouk, M.S. Wrighton, J. Phys. Chem. 91 (1987) 648.

[6] P. Hoyer, H. Weller, J. Phys. Chem. 99 (1995) 14096.

[7] E.A. Meulenkamp, J. Phys. Chem. B 103 (1999) 7831.
[8] P.E. De Jongh, E.A. Meulenkamp, D. Vanmaekelbergh, et al., J. Phys. Chem. B 104 (2000) 7686.

[9] A.L. Roest, J.J. Kelly, D. Vanmaekelbergh, et al., Phys. Rev. Lett. 89 (2002) 036801.

[10] M. Shim, P. Guyot-Sionnest, Nature 407 (2000) 981.

[11] C. Wang, M. Shim, P. Guyot-Sionnest, Science (Washington, DC, U.S.) 291 (2001) 2390.

[12] D. Yu, C. Wang, P. Guyot-Sionnest, Science 300 (2003) 1277.

[13] B.L. Wehrenberg, P. Guyot-Sionnest, J. Am. Chem. Soc. (2003).

[14] A. Germeau, A.L. Roest, D. Vanmaekelbergh, et al., Phys. Rev. Lett. 90 (2003) 097401/1

[15] A.L. Roest, J.J. Kelly, D. Vanmaekelbergh, Appl. Phys. Lett. 83 (2003) 5530 .

[16] A.L. Roest, A.J. Houtepen, J.J. Kelly, et al., Faraday Discuss. 125 (2003) 55 .

[17] A.J. Houtepen, D. Vanmaekelbergh, J. Phys. Chem. B 109 (2005) 19634.

[18] I. Heller, J. Kong, H.A. Heering, et al., Nano Lett. 5 (2005) 137.

[19] I. Heller, J. Kong, K.A. Williams, et al., J. Am. Chem. Soc. 128 (2006) 7353.

[20] M. Bockrath, J. Hone, A. Zettl, et al., Phys. Rev. B 61 (2000) 10606.

[21] S. Rosenblatt, Y. Yaish, J. Park, et al., Nano Lett. 2 (2002) 869.

[22] L. Larrimore, S. Nad, X.J. Zhou, et al., Nano Lett. 6 (2006) 1329.

[23] D.I. Gittins, D. Bethell, D.J. Schiffrin, et al., Nature (London) 408 (2000) 67.

[24] C.D. Jaeger, H. Gerischer, W. Kautek, Berichte der Bunsen-Gesellschaft 86 (1982) 20.

[25] H. Benisty, J.N. Chazalviel, J. Electrochem. Soc. 140 (1993) 1949.

[26] R. van de Krol, A. Goossens, J. Schoonman, J. Phys. Chem. B 103 (1999) 7151.

[27] M. Shibuya, T. Nishina, T. Matsue, et al., J. Electrochem. Soc. 143 (1996) 3157.

[28] R. van de Krol, A. Goossens, E.A. Meulenkamp, J. Appl. Phys. 90 (2001) 2235.

[29] D. Cummins, G. Boschloo, M. Ryan, et al., J. Phys. Chem. B 104 (2000) 11449.

[30] C.J. Wang, M. Shim, P. Guyot-Sionnest, Appl. Phys. Lett. 80 (2002) 4.

[31] P. Guyot-Sionnest, C. Wang, J. Phys. Chem. B 107 (2003) 7355.

[32] T.M. Day, N.R. Wilson, J.V. Macpherson, J. Am. Chem. Soc. 126 (2004) 16724.

[33] H. Shirakawa, E.J. Louis, A.G. MacDiarmid, et al., Chem. Soc., Chem. Commun. 578 (1977).

[34] C.K. Chiang, C.R. Fincher, Y.W. Park, et al., Phys. Rev. Lett. 39 (1977) 1098.

[35] J.J.M. Halls, C.A. Walsh, N.C. Greenham, et al., Nature (London) 376 (1995) 498.

[36] G. Yu, J. Gao, J.C. Hummelen, et al., Science (Washington, DC) 270 (1995) 1789.

[37] P.K.H. Ho, J.-S. Kim, J.H. Burroughes, et al., Nature (London) 404 (2000) 481.

[38] E.W. Paul, A.J. Ricco, M.S. Wrighton, J. Phys. Chem. 89 (1985) 1441.

[39] R. van de Krol, A. Goossens, E.A. Meulenkamp, J. Electrochem. Soc. 146 (1999) 3150.

[40] R. van de Krol, A. Goossens, E.A. Meulenkamp, J. Electrochem. Soc. 146 (1999) 3150.

[41] E.S. Kooij, A.T.M. van Gogh, R. Griessen, J. Electrochem. Soc. 146 (1999) 2990.

[42] S.J. Van der Molen, D.G. Nagengast, A.T.M. van Gogh, et al., Phys. Rev. B 63 (2001) 235116.

[43] J.N. Huiberts, R. Griessen, J.H. Rector, et al., Nature 380 (1996) 231.

[44] P.H.L. Notten, R. Kremers, R. Griessen, J. Electrochem. Soc. 143 (1996) 3348.

[45] A.P. Alivisatos, J. Phys. Chem. 100 (1996) 13226.

[46] C.B. Murray, D.J. Norris, M.G. Bawendi, J. Am. Chem. Soc. 115 (1993) 8706.

[47] C.B. Murray, C.R. Kagan, M.G. Bawendi, Science 270 (1995) 1335.

[48] R. Rossetti, S. Nakahara, L.E. Brus, J. Chem. Phys. 79 (1983) 1086.

[49] E.P.A.M. Bakkers, Z. Hens, A. Zunger, et al., Nano Lett. 1 (2001) 551.

[50] L.E. Brus, Jerusalem Symp. Quant. Chem. Biochem. 17 (1984) 431. 
[51] A.J. Houtepen, R. Koole, D.L. Vanmaekelbergh, et al., J. Am. Chem. Soc. 128 (2006) 6792.

[52] B.L. Wehrenberg, C. Wang, P. Guyot-Sionnest, J. Phys. Chem. B 106 (2002) 10634.

[53] D. Vanmaekelbergh, A.L. Roest, A. Germeau, et al., Phys. Rev. Lett. 91 (2003).
[54] D. Yu, C. Wang, B.L. Wehrenberg, et al., Preprint Archive, Condensed Matter, vol. 1, Los Alamos National Laboratory, 2004.

[55] D.V. Talapin, C.B. Murray, Science 310 (2005) 86.

[56] C. Wang, B.L. Wehrenberg, C.Y. Woo, et al., J. Phys. Chem. B, ASAP Article, 2004. 10.1021/jp0489830 S1089. 